\title{
A Review on Gene Therapy
}

\author{
R. S. Rakhi Menon
}

\begin{abstract}
Gene therapy, also often referred to as 'gene transfer,' uses carriers called 'vectors' to deliver genes to tissues where they are needed. Gene therapy is composed of two categories: somatic gene therapy and germ line gene therapy. In somatic gene therapy, therapeutic genes are introduced to the diseased cells of a patient in hopes that they will genetically alter them to function normally. In germ line gene therapy, therapeutic genes are introduced to reproductive cells (egg and sperm cells) to prevent the manifestation of a genetic disorder before the patient is born.
\end{abstract}

Keywords: Gene Therapy, Somatic Gene Therapy

\section{Introduction}

Current therapies for most human genetic diseases are inadequate. In response to the need for effective treatments, modern molecular genetics is providing tools for an unprecedented new approach to disease treatment through an attack directly on mutant gene. (T Friedmann- Progress toward human gene therapy).

Gene therapy, also often referred to as 'gene transfer,' uses carriers called 'vectors' to deliver genes to tissues where they are needed.

Gene therapy is composed of two categories: somatic gene therapy and germ line gene therapy.

In somatic gene therapy, therapeutic genes are introduced to the diseased cells of a patient in hopes that they will genetically alter them to function normally.

In germ line gene therapy, therapeutic genes are introduced to reproductive cells (egg and sperm cells) to prevent the manifestation of a genetic disorder before the patient is born.

Gene therapy is an experimental technique that uses genes to treat or prevent disease. In the future, this technique may allow doctors to treat a disorder by inserting a gene into a patient's cells instead of using drugs or surgery. Researchers are testing several approaches to gene therapy, including:

- Replacing a mutated gene that causes disease with a healthy copy of the gene.

- Inactivating, or "knocking out," a mutated gene that is functioning improperly.

- Introducing a new gene into the body to help fight a disease.

\section{Gene Therapy for Neurological Advancement}

Neurological disorders are among the most difficult to treat with traditional pharmacological approaches due to the complexity of the nervous system and the physical barriers (such as the blood-brain barrier, that limit the distribution of many compounds into the central nervous system (CNS) after peripheral administration.
Vector-mediated gene transfer is an alternative strategy to treat CNS disorders that holds much promise, especially for monogenic diseases.

\section{Gene Delivery Vectors}

Viruses have the natural ability to deliver genetic material to cells, which makes them an excellent vector for gene delivery. Lentivirus, Herpes Simplex Virus, Adenovirus and Adeno-Associated virus (AAV) are some of the commonly used vectors for gene delivery to the CNS .(1)

\section{Current State of Clinical Research}

Two primary strategies have emerged as a means to advance gene therapy into the clinical arena.

In vivo

Gene transfer focuses on using viral or chemical reagents to deliver genes directly to patients through a single injection.

Ex vivo

Gene transfer relies on externally transducing cultured cells with a therapeutic vector and injecting them back into patients (2)

\section{Advancements in Vector Technology for In Vivo Gene Transfer}

An ideal vector would provide widespread distribution of the transgene, large packaging capacity, stable gene expression, high transduction efficiency, target cell specificity, and low immunological responses in order to overcome current limitations. To increase the specificity of the viral vector, known ligands for target cells can be incorporated on the capsid.

\section{Advancements in Ex Vivo Gene Transfer}

Successful treatment options for lysosomal storage diseases include enzyme replacement therapy, hematopoietic cell transplantation, or a combination of the two as seen in Hurler's syndrome.

The main problem with ERT is the efficacy of the enzyme to cross the BBB which limits the access of systemically injected enzyme. Allogeneic HCT can be used to overcome this. Hematopoietic cells, such as activated lymphocytes, monocytes, and microglial precursors, are able to cross the $\mathrm{BBB}$ and can be used to deliver enzymes to the CNS (3).

Volume 6 Issue 7, July 2017 


\section{International Journal of Science and Research (IJSR) \\ ISSN (Online): 2319-7064 \\ Index Copernicus Value (2015): 78.96 | Impact Factor (2015): 6.391}

\section{Summary}

Monogenic CNS disorders, especially LSDs that can benefit from cross-correction, are particularly amenable to this approach. Clinical trials spanning the prior decade have shown positive effects resulting from gene transfer, but the benefit to the patients has been relatively modest. The vector technology, both forin vivoand ex vivogene transfer, has advanced rapidly in the last few years and new approaches have been developed to significantly improve the scope and efficiency of gene transfer.

\section{Gene Therapy For Adenosine Deaminase Deficient Severe Combined Immune Deficiency}

Adenosine deaminase is an enzyme for purine metabolism and is essential for lymphocyte development, its survival, and function. Deficiency of ADA is an autosomal recessive inherited disorder and can result in severe combined immune deficiency (SCID). Treatment of choice for ADA-Deficient SCID is a hematopoietic stem cell (HSC) transplantation from an infected, HLA-match sibling. (4)

Current clinical results indicate that $(a)$ both umbilical cord blood and neonatal bone marrow HSC can be transduced with murine retroviral-based vectors, $(b)$ the transduced HSC can engraft in non-myeloablated patients, $(c)$ the frequency of HSC transduction/engraftment is low $(1 / 10,000),(d)$ an in vivo selective advantage can exist for transduced $\mathrm{T}$ lymphoid progeny, and $(e)$ the transduced ADA gene is not expressed in non-dividing $\mathrm{T}$ lymphocytes. \{Robertson Parkman' Kenneth Weinberg ${ }^{1}$ Gay Crooks $^{1}$ Jan Nolta ${ }^{1}$ Neena Kapoor ${ }^{1}$ and Donald Kohn ${ }^{1}$ Gene Therapy for Adenosine Deaminase Deficiency). Gene therapy, combined with reduced-intensity conditioning, is a safe and effective treatment for SCID in patients with ADA deficiency. Annual Review of Medicine). Gene therapy, combined with reduced-intensity conditioning, is a safe and effective treatment for SCID in patients with ADA deficiency. (5)

\section{Gene Therapy in Cardio Vascular Disease}

The field of cardiovascular gene transfer has developed rapidly during the past 5 years. Important advances have been made in vector development, in vivo gene delivery, and definition of potential therapeutic targets. (6)

\section{a) Reterovoral Vector}

As vascular cells are accessible through the blood stream, percutaneous site, specific gene delivery was developed for local arterial segment. Retro viral vectors were initially used in in vivo vascular gene transfer. (7)

\section{b) Adenoviral Vector}

To improve efficacy of direct gene transfer into arterial, research are now focused on adenoviral vectors. Adenovirus serotype $2 \& 5$ have been developed as viral vector for gene transfer (8).

\section{c) Adenovirus Augmented, Receptor Mediated Gene Delivery System}

Inactivated adenovirus is complexed with a receptor ligand that facilitates entry of DNA into cell(.Cotten M, Wagner E, Zatloukal K, Phillips S, Curiel DT, Birnstiel ML. Highefficiency receptor-mediated delivery of small and large (48 kilo base) gene constructs using the endosome-disruption activity of defective or chemically inactivated adenovirus particles. Proc Natl Acad Sci U S A. 1992; 89:6094-6098.) The virus is coupled to a ligand such as transferrin. The transferrin ligand binds to a transferrin receptor in a cell, and the transferrin viral polylysine DNA complex enters the cell by receptor-mediated endocytosis. The inactivated adenovirus functions to disrupt lysosomes in the host cell, reducing DNA degradation and releasing DNA into the cytoplasm (9).

\section{Conclusion}

Gene therapy have good implications for future generations that are known to have detrimental genes. The development of serious and unfavorable inherited genetic diseases could be prevented before birth and eliminated in the following generations. The future of gene therapy right now to me looks promising, but there is a lot of work to be done.

\section{References}

[1] Sahana Nagabhushan Kalburi:Recent Gene Therapy Advancements for Neurological Diseases

[2] Suhr and Gage, 1993

[3] Asheuer et al., 2004; Priller et al., 2001

[4] gene therapy for adenosine deaminase-deficient severe combined immune deficiency: clinical comparison of retroviral vectors and treatment plans Fabio candotti, Kit L Shaw, Linda Muul, Denise Carbonaro, Robert Sokolic, Christopher Choi, Sheperd H Schurman, Elizabeth Garabedian, Chimene Kesserwan, G Jayashree Jagadeesh, Pei-Yu Fu, Eric Gschweng, Aaron Cooper, John F Tisdale, Kenneth I Weinberg, Gay M Crooks, Neena kapoor, Ami Shah, Hisham Abdel-Azim, Xiao-Jin Yu, Monika Smogorzewska, Alan S Wayne, Howard M Rosenblatt, Clara M Davis, Celin Hanson, Radha G Rishi, Xiaoyan Wang, David Gjertson, Otto O Yang, Arumugm Balamurugan, Gerhard Bauer, Joanna A Ierland. Barbara C Engel, Gregory M Podsakoff, Michael Blaese, Robertson Pakman And Donald B Khon.

[5] Gene Therapy for Immunodeficiency Due to Adenosine Deaminase Deficiency

[6] Alessandro Aiuti, M.D., Ph.D., Federica Cattaneo, M.D., Stefania Galimberti, Ph.D., Ulrike Benninghoff, M.D., Barbara Cassani, Ph.D., Luciano Callegaro, R.N., Samantha Scaramuzza, Ph.D., Grazia Andolfi, Massimiliano Mirolo, B.Sc., Immacolata Brigida, B.Sc., Antonella Tabucchi, Ph.D., Filippo Carlucci, Ph.D., Martha Eibl, M.D., Memet Aker, M.D., Shimon Slavin, M.D., Hamoud Al-Mousa, M.D., Abdulaziz Al Ghonaium, M.D., Alina Ferster, M.D., Andrea Duppenthaler, M.D., Luigi Notarangelo, M.D., Uwe 


\section{International Journal of Science and Research (IJSR) \\ ISSN (Online): 2319-7064}

Index Copernicus Value (2015): 78.96 | Impact Factor (2015): 6.391

Wintergerst, M.D., Rebecca H. Buckley, M.D., Marco

Bregni, M.D., Sarah Marktel, M.D., Maria Grazia

Valsecchi, Ph.D., Paolo Rossi, M.D., Fabio Ciceri, M.D.,

Roberto Miniero, M.D., Claudio Bordignon, M.D., and Maria-Grazia Roncarolo, M.D.

[7] N Engl J Med 2009; 360:447-458January 29, 2009DOI: 10.1056/NEJMoa0805817

[8] GENE THERAPY FOR CARDIO VASCULAR DISEASE-Elizabeth G. Nabel, MD

[9] Nabel EG, Plautz G, Boyce FM, Stanley JC, Nabel GJ. Recombinant gene expression in vivo within endothelial cells of the arterial wall. Science.1989;244:1342-1344

[10] Berkner KL. Expression of heterologous sequences in adenoviral vectors. Curr Top Microbial Immunol.1992; 58:39-66.)

[11].( Curiel DT, Agarwal S, Wagner E, Cotten M. Adenovirus enhancement of transferrin-polylysinemediated gene delivery. Proc Natl Acad Sci U S A.1991; 88:8850-8854. 